\title{
Kinematic parameters of throwing performance in patients with schizophrenia using a markerless motion capture system
}

\author{
Fátima Sá ${ }^{1}$, António Marques ${ }^{1}$, Nuno B. F. Rocha ${ }^{1}$, Maria J. Trigueiro ${ }^{1}$, Carlos Campos ${ }^{1}$, \& Johannes Schröder ${ }^{2}$ \\ ${ }_{1}$ Polytechnic Institute of Porto, School of Allied Health Sciences, Porto, Portugal and ${ }^{2}$ Section of Geriatric Psychiatry, Department of Psychiatry, \\ University of Heidelberg, Heidelberg, Germany
}

\begin{abstract}
Motor dysfunction is consistently reported but understudied in schizophrenia. It has been hypothesized that this abnormality may reflect a neuro-developmental disorder underlying this illness. The main goal of this study was to analyze movement patterns used by participants with schizophrenia and healthy controls during overarm throwing performance, using a markerless motion capture system. Thirteen schizophrenia patients and 16 healthy control patients performed the overarm throwing task in a markerless motion capture system. Participants were also examined for the presence of motor neurological soft signs (mNSS) using the Brief Motor Scale. Schizophrenia patients demonstrated a less developed movement pattern with low individualization of components compared to healthy controls. The schizophrenia group also displayed a higher incidence of mNSS. The presence of a less mature movement pattern can be an indicator of neuro-immaturity and a marker for atypical neurological development in schizophrenia. Our findings support the understanding of motor dysfunction as an intrinsic part of the disorder of schizophrenia.
\end{abstract}

Keywords Markerless motion analysis, motor abnormalities, motor immaturity, neurological soft signs, schizophrenia

\section{Introduction}

Motor abnormalities are frequent in schizophrenia and have been reported since early descriptions of the illness (Manschreck 1992; Kent et al. 2012). These deficits have assumed particular clinical importance in schizophrenia and undoubtedly have an impact on long-term outcomes, affecting social, academic, emotional, and daily functioning (Manschreck 1992; Putzhammer and Klein 2006; Varlet et al. 2012).

After the introduction of antipsychotic pharmacology, motor symptoms were progressively attributed to the side effects of the treatment (Manschreck 1992). There is evidence which supports the role of antidopaminergic intervention in the appearance of motor symptoms, with a crucial role of D2 receptor blockade (Veselinović et al. 2011). Although they can be caused by antipsychotic medication, there is growing evidence that motor deficits can predate the onset of the psychosis and can be referred as a primary symptom of the disorder itself (Wolff and O'Driscoll 1999). Longitudinal studies have also demonstrated that children who later develop schizophrenia spectrum disorders had displayed late acquisition of milestones and poor coordination during the first few years of life (Erlenmeyer-Kimling et al. 2000;
Cannon et al. 2002; Welham et al. 2009; Clarke et al. 2011; Moilanen 2011). These results suggest that motor deficits may serve as risk markers for the illness and are consistent with the neuro-developmental model of schizophrenia (Wolff and O'Driscoll 1999; Tigges et al. 2000; Walther and Strik 2012).

Motor disturbances in schizophrenia have been widely examined within the category of neurological soft signs (NSS), which refer to subtle abnormalities in motor coordination, sensory integration, and sequencing of complex motor movements (Buchanan and Heinrichs 1989). The presence of these subtle neurological abnormalities indicates a neuro-immaturity and tends to disappear with age (Blondis et al. 1990; Gillberg and Kadesjo 2003; Martins et al. 2013). NSS have been reported frequently in schizophrenia patients, even in first episode psychosis and drug-naive patients, as well as in their unaffected first-degree relatives, showing correlations with severity and persistence of psychopathological symptoms, poor social functioning, and with a range of neuro-cognitive abnormalities (Bombin et al. 2005; Varambally et al. 2012).

Despite the high prevalence of motor deficits in schizophrenia (78-97\% prevalence of NSS in first episode medicationnaive patients), investigations with quantitative measurements remained scarce (Walther and Strik 2012). There are some studies that employed kinematic analysis of repetitive hand movements, such as diadochokinesis and finger tapping (that are subtests included in the NSS rating scales), to evaluate 
motor function in schizophrenia. The results showed that schizophrenia patients have difficulty performing these tasks mainly with a reduction of regularity, amplitude, and peak velocity (Fuller and Jahanshahi 1999; Tigges et al. 2000; Klausmann 2003; Putzhammer et al. 2005a, 2005b). Other studies also using kinematic analysis of motion revealed alterations in gait velocity by a decrease in stride length (Putzhammer and Klein 2006) and alterations in the postural sway area (Kent et al. 2012). However, little is known about kinematic parameters of fundamental motor behaviors frequently used in daily life. Overarm throwing has a specific observable motor pattern and is commonly used in daily life (Marques and Catenassi 2005; Haywood and Getchell 2009), which was why this task was chosen for the present study. During performance of this task, the whole body is involved, requiring sequential actions of all segments and coordination of fine and gross movements (Haywood and Getchell 2009). Control of overarm throwing develops through several stages until 7-9 years of age, where the movement pattern reaches a more mature stage, which is maintained throughout adulthood (Gallahue and Ozmun 2003). According to Roberton and Halverson (1984), the development sequences of overarm throwing are best described using a component approach that comprises developmental steps of increasing maturity for the following components: humerus, forearm, trunk, and feet. The mature motor pattern of overarm throwing is characterized mainly by a strong coupling among components where a differentiated rotation between the trunk and pelvis occurred as well as a downward backswing movement in the upper arm for an increase in the strength of the release.

The main goal of this study then was to analyze movement patterns used by participants with schizophrenia and healthy controls during overarm throwing performance, using a markerless motion capture system.

\section{Methods}

\section{Participants}

Thirteen clinically stable outpatients with schizophrenia (Group I) and 16 healthy controls (Group II) were enrolled in the study. Patients were previously diagnosed according to the Structural Clinical Interview for DSM-IV and were recruited from two sites: Clínica de Psiquiatria e Saúde Mental do Centro Hospitalar de S. João (CHSJ), Porto, Portugal and Associação Nova Aurora na Reabilitação Psicossocial (ANARP), Porto, Portugal. Participants from Group II were volunteers who freely agreed to participate in this study. The two groups were matched for gender, age, body mass index, and education level. Exclusion criteria for all participants comprised a history of neurological illness and current substance abuse or dependence. For Group II, additional exclusion criteria were: personal history of Axis I/II disorders or psychiatric history in first or seconddegree relatives.

No statistical differences were found between the two groups regarding socio-demographic variables and premorbid intelligence, according to the score from the Vocabulary (Table I). Group I had more motor neurological soft signs (mNSS) compared to Group II. All participants were
Table I. Summary of participant characteristics.

\begin{tabular}{lccc}
\hline & \multicolumn{2}{c}{ Group } & \\
\cline { 2 - 3 } & \multicolumn{1}{c}{$\mathrm{I}(n=13)$} & $\mathrm{II}(n=16)$ & \\
& $\mathrm{M}(\mathrm{SD})$ & $\mathrm{M}(\mathrm{SD})$ & $p$ \\
\hline Gender (F/M) & $1 / 12$ & $2 / 14$ & $1.00^{\mathrm{a}}$ \\
Age (years) & $44.38(5.78)$ & $41.38(8.57)$ & $0.49^{\mathrm{b}}$ \\
Education level (years) & $11.46(3.82)$ & $11.13(4.47)$ & $0.87^{\mathrm{b}}$ \\
Body mass index & $26.39(2.82)$ & $26.31(3.39)$ & $0.73^{\mathrm{c}}$ \\
Vocabulary subtest score & $11.31(3.04)$ & $10.00(1.75)$ & $0.09^{\mathrm{b}}$ \\
BMS ${ }^{\mathrm{d}}$ score & $6.12(3.42)$ & $0.56(0.73)$ & $\leq 0.001^{\mathrm{b}}$ \\
$\quad$ Motor Coordination Subscale & $3.46(1.99)$ & $0.34(0.54)$ & $\leq 0.001^{\mathrm{b}}$ \\
$\quad$ Motor Sequencing Subscale & $2.65(2.09)$ & $0.22(0.31)$ & $\leq 0.001^{\mathrm{b}}$ \\
Chlorpromazine equivalent & $414.94(285.83)$ & - & \\
$\quad$ dose (mg/day) & & & \\
PANSS ${ }^{\mathrm{b}}$ total score & $65.69(28.05)$ & - & \\
$\quad$ Positive Subscale & $14.77(8.43)$ & & \\
$\quad$ Negative Subscale & $18.00(7.30)$ & & \\
$\quad$ General Subscale & $35.15(14.96)$ & & \\
\hline
\end{tabular}

${ }^{\mathrm{a}}$ Fisher's exact test.

${ }^{\mathrm{b}}$ Mann-Whitney.

${ }^{\mathrm{c}}$ Independent samples $t$-test.

${ }^{\mathrm{d}} \mathrm{BMS}=$ Brief Motor Scale.

${ }^{\mathrm{e}} \mathrm{PANSS}=$ Positive and Negative Syndrome Scale.

right-handed. Participants from Group I were taking antipsychotics and the mean chlorpromazine equivalent was calculated (Woods 2003). This study was approved by a local ethics committee (CHSJ) and by the directive board of ANARP. Written informed consent was obtained from all participants prior to study participation according to the Helsinki Declaration.

\section{Instruments}

Kinematic parameters of overarm throwing were measured using a markerless motion capture system-BioStage ${ }^{\mathrm{TM}}$ (Organic Motion, New York, USA). This is an advanced optical tracking system that instantly generates real-time 3D data of all the body and enables a comprehensive analysis of human motion. BioStage ${ }^{\mathrm{TM}}$ acquires motion in a noninvasive manner with 14 monochromatic cameras $(752 \times 480$ resolution) using a scan space of $5 \times 4 \times 2.5 \mathrm{~m}^{3}$ and reflective-canvas walls, without the need for a body suit or movement sensors. The overarm throwing movement was recorded at $60 \mathrm{~Hz}$ (obtained at 60 frames per second) and motion data were analyzed using the MotionMonitor ${ }^{\mathrm{TM}}$ (Innovative Sports Training, Chicago, IL, USA), a software program for analyzing biomechanical motion (Organic Motion 2012).

The presence of mNSS was examined using the Brief Motor Scale (BMS) which comprises two subscales: Motor Coordination and Motor Sequencing (Jahn et al. 2006). The Vocabulary subtest, from the WAIS-III (Wechsler 1997), was used as a measure of premorbid intelligence for all participants. The severity of schizophrenia-related symptoms was examined using the Positive and Negative Syndrome Scale (PANSS) (Kay et al. 1987).

\section{Procedures}

Prior to the movement capture using the BioStage Organic Motion $^{\mathrm{TM}}$ system, all procedures were explained and 


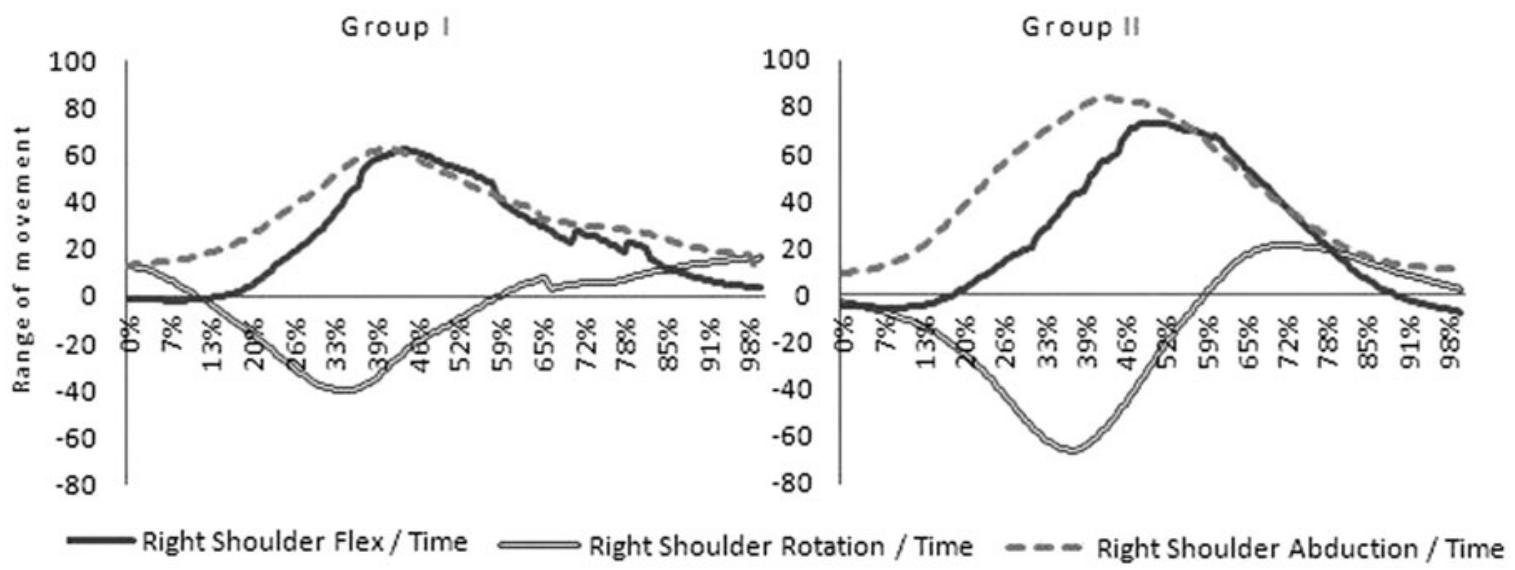

Figure 1. Right shoulder movement for Group I (shown on the left) and Group II (shown on the right).

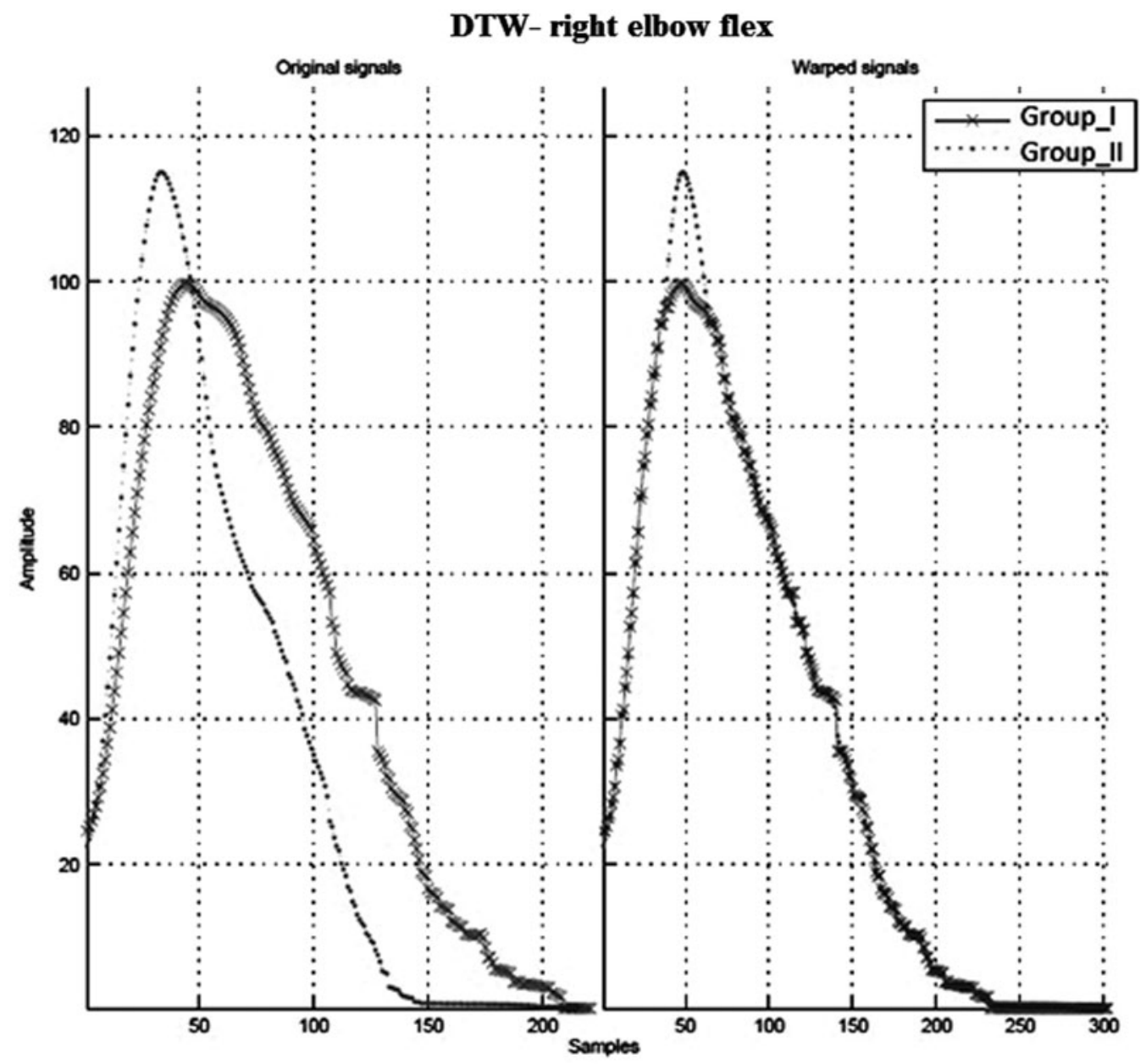

Figure 2. Dynamic time warping (DTW) algorithm computed for right elbow flex motion for both groups.

demonstrated by one researcher. To obtain system calibration, participants were asked to stand in bare feet at the center of the scan space, with their legs apart and their arms parallel to the floor. After this, they were asked to throw a tennis ball at the target, as naturally as possible, with their arm raised above their shoulder from a starting position with their arms along their body. The conditions of the overarm throwing assessment were based on Subtest 5 (item 5) of the Bruininks-Oseretsky Test of Motor Proficiency (Bruininks 1978). According to the administration instructions, the subject throws a tennis ball overarm at the target with his or her preferred hand from a distance of 5 feet $(1.5 \mathrm{~m})$ and 


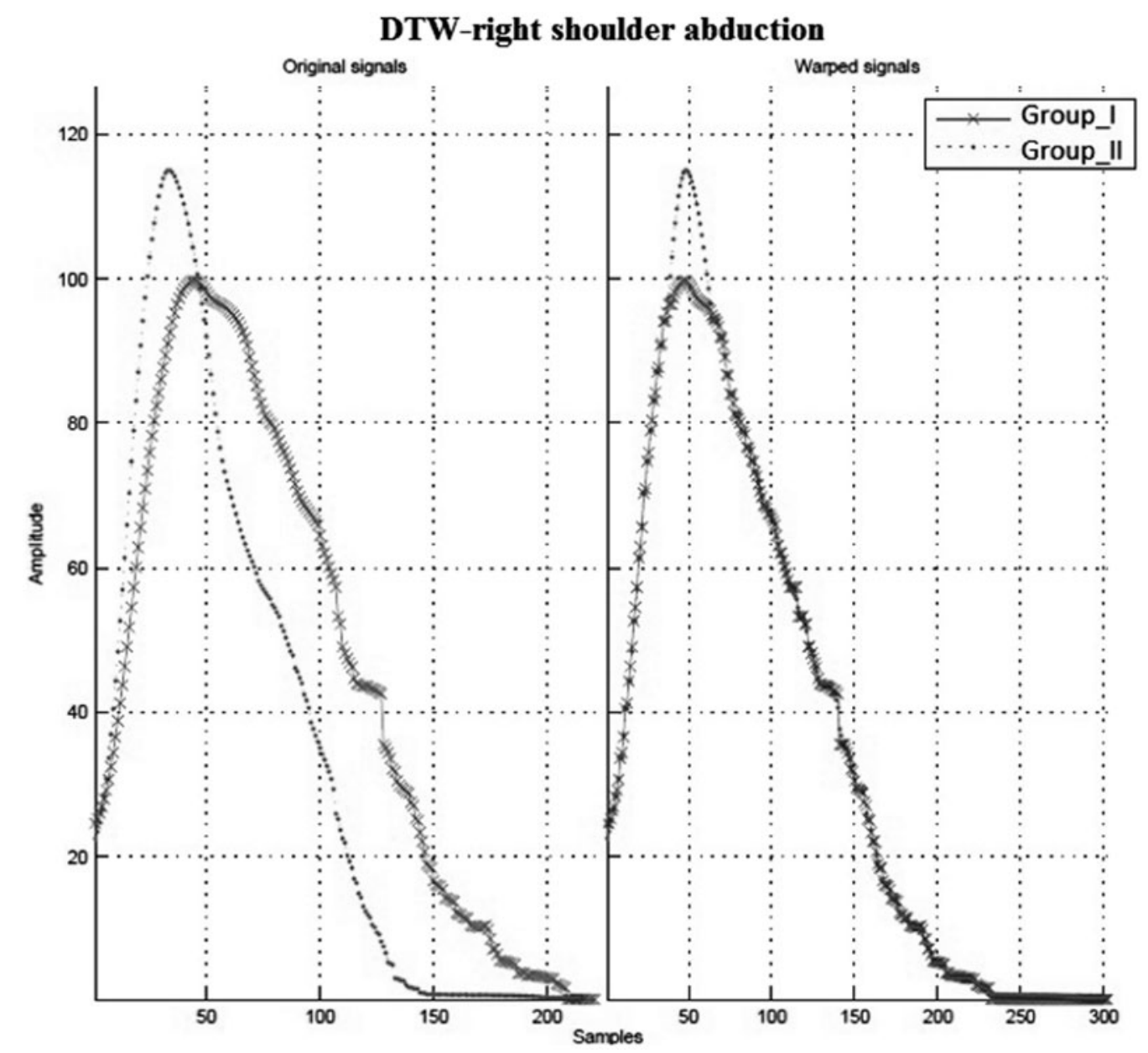

Figure 3. Dynamic time warping (DTW) algorithm computed for right shoulder abduction motion for both groups.

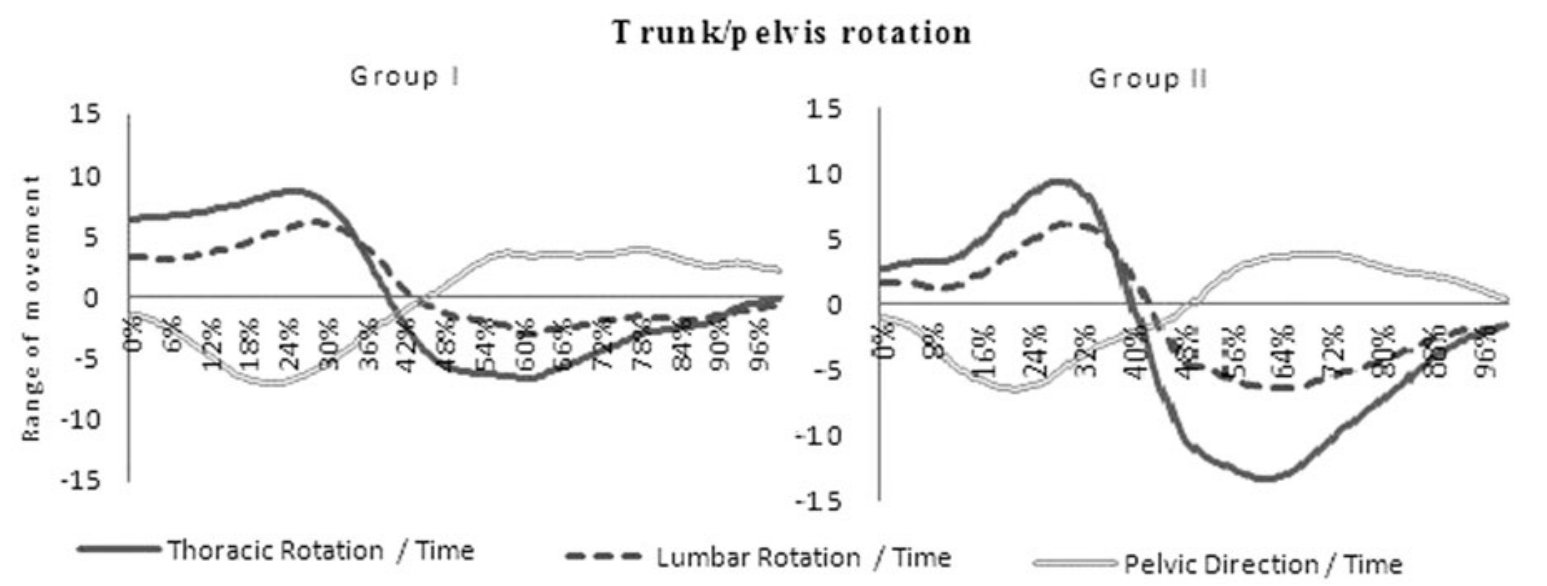

Figure 4. Trunk and pelvic rotation movements for Group I (shown on the left) and Group II (shown on the right).

repeats this 5 times. This test was selected to ensure a parameterized and valid assessment of motor behavior of all participants, and also because this test is considered a discriminative and evaluative measure for characterizing motor performance. The original subtest rating is based on the number of target hits, thereby there are no normative values regarding range of motion for this task.

\section{Data analysis}

The 3D motion data were analyzed using the Motion Monitor $^{\mathrm{TM}}$ software. The movement parameter selected was the range of motion of all body segments. Motion data from participants were imported to Microsoft Office Excel 2007 and were log-normalized at $100 \%$ using a math-routine, to 


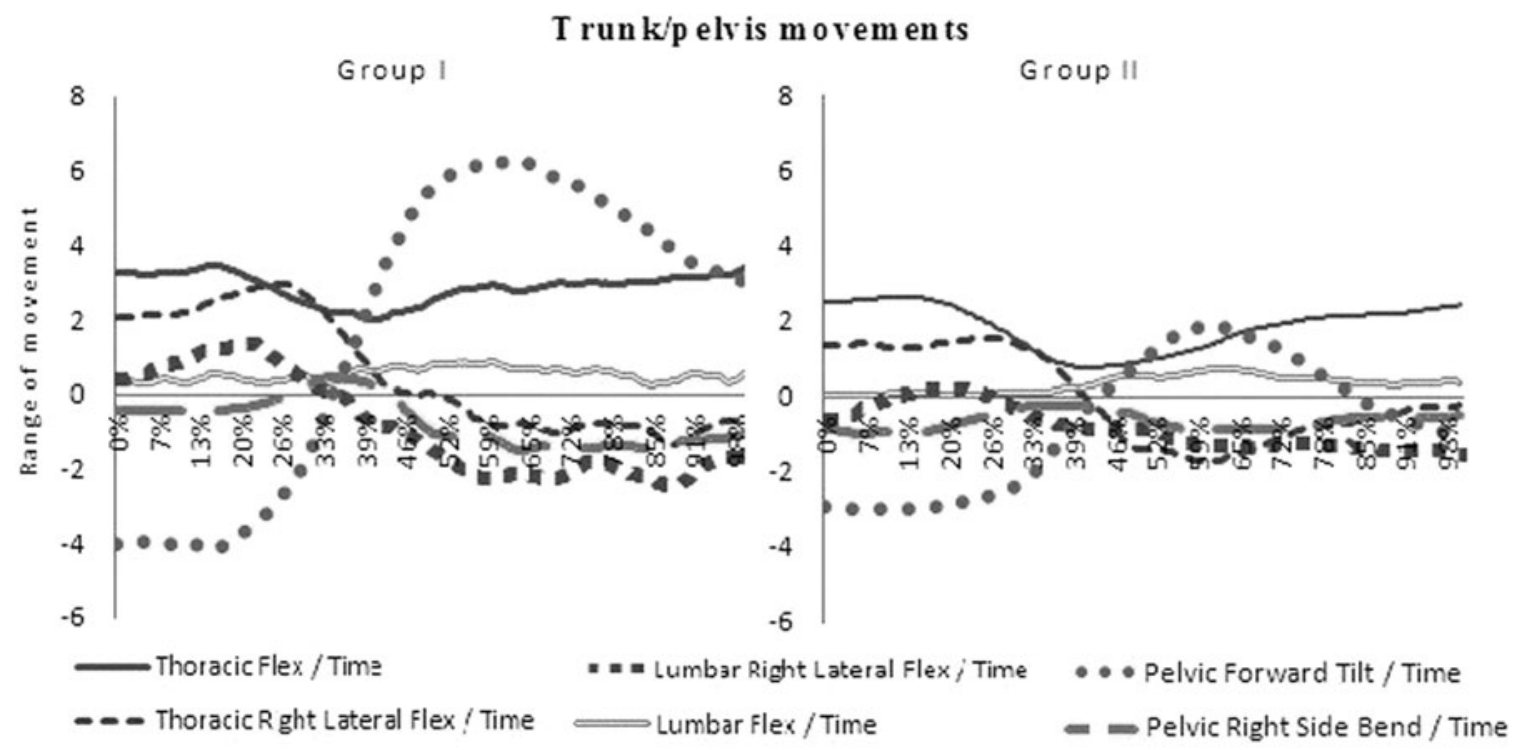

Figure 5. Trunk and pelvic inclination and flex movements for Group I (shown on the left) and Group II (shown on the right).

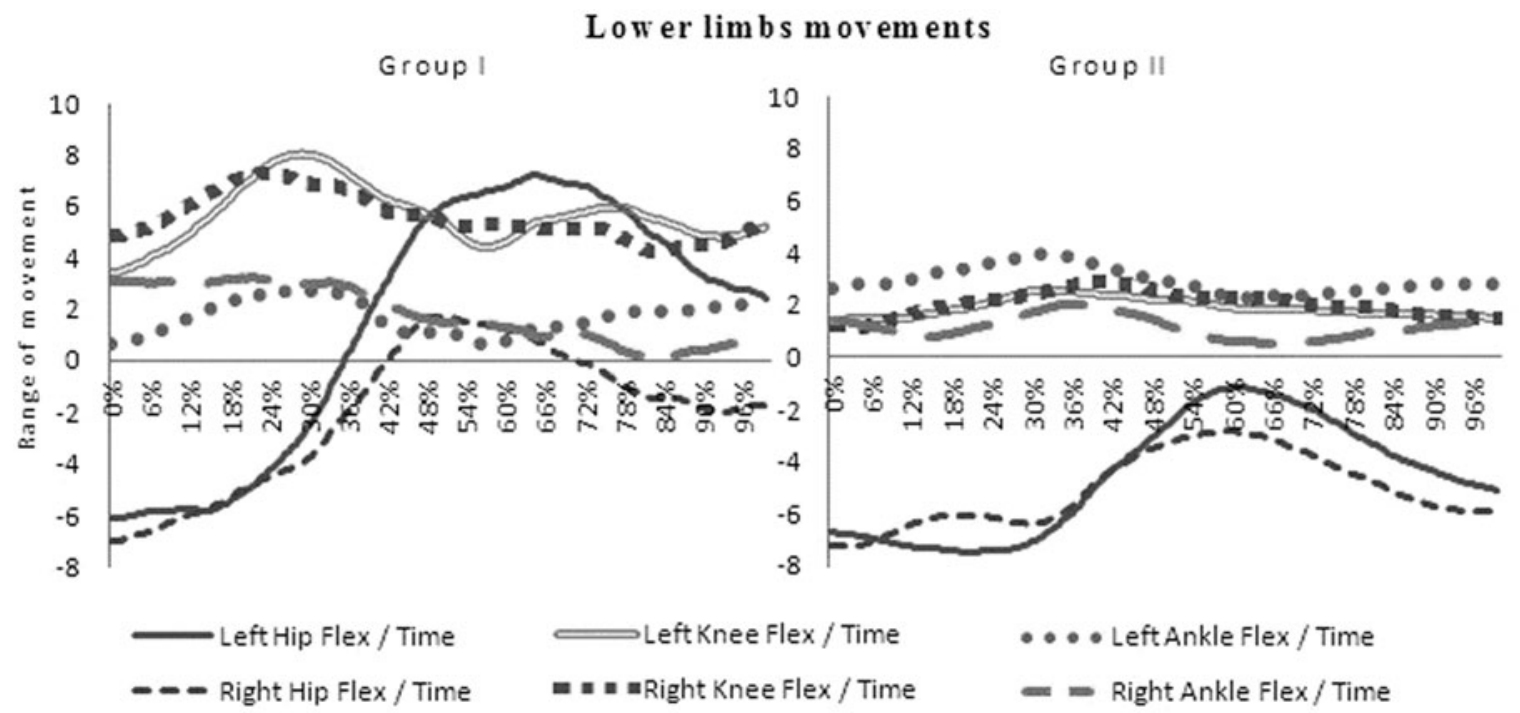

Figure 6. Hip, knee, and ankle flex movements for Group I (shown on the left) and Group II (shown on the right).

ensure that the size of all samples was the same $(n=200)$. The mean values of the range of motion were calculated for all body segments and graphs were plotted representing the motion of each joint over time for each group of participants. After this, data were analyzed qualitatively comparing the average range of motion and the trajectory of movements of each body component between each group. This analysis allows an understanding of which motor pattern of overarm throwing was recruited by participants with schizophrenia and the healthy controls.

Data were also analyzed quantitatively by estimating the duration of the overarm throwing movement and comparing differences between both groups' averages. The root mean square error (RMSE) was applied to compare temporal signals of movement which gives the average difference between two time series. To solve the normalization problem, a technique known as dynamic time warping (DTW) was used. DTW uses dynamic programming to find the best non- linear mapping between the two signals by minimizing the distance between them (Sakoe and Chiba 1978; Müller 2007). DTW was applied to all pairs of samples in MATLAB ${ }^{\circledR}$ R2010b.

Statistical analyses were performed using SPSS 16.0 Statistics. The differences between groups were analyzed by the independent samples $t$-test or the Mann-Whitney test (when samples do not meet the assumption of normality) for quantitative variables, such as: socio-demographic characteristics, total scores of clinical assessments, and kinematic parameters of motion (total movement duration). In the case of nominal variables (such as gender), group differences were verified using Fisher's exact test.

RMSE is used to measure the differences between two things that may vary, neither of which is accepted as the standard, as when measuring the average distance between two random vectors (Kenney and Keeping 1962). The RMSE is the square root of the variance, known as the standard error, 


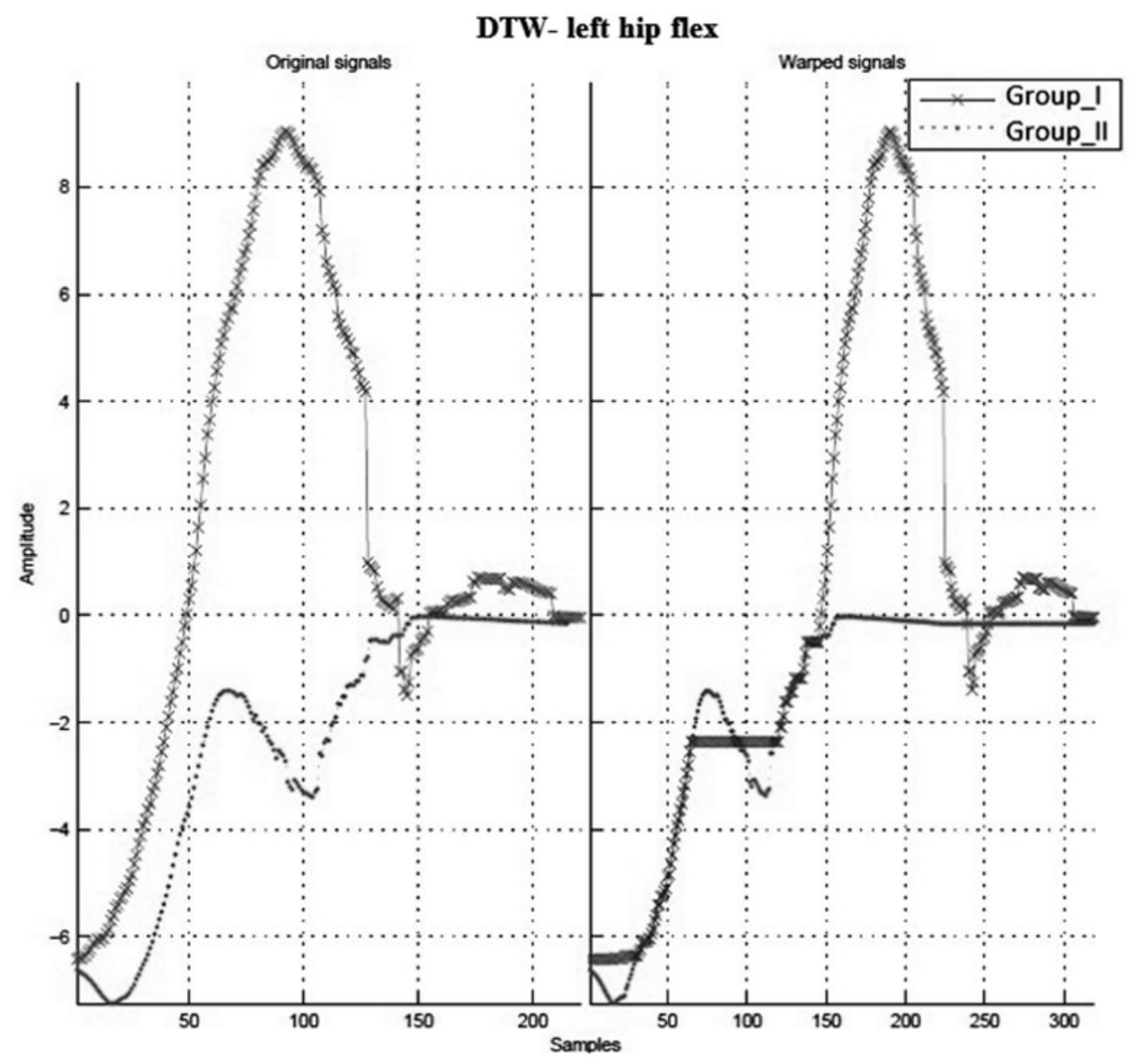

Figure 7. Dynamic time warping (DTW) algorithm computed for left hip flex motion for both groups.

and it represents the average distance between the two curves that indicate the average values for several movements, for each pair Group I/Group II. This measure is expressed in the same units as the data and is representative of the size of a "typical" error. There is no absolute criterion for a "good" value of RMSE: it depends on the units in which the variable is measured.

\section{Results}

The kinematic analysis of the movements performed by participants shows some differences between groups. Although all the participants exhibited similar patterns in the movements performed with the upper limbs, it can be noted that Group II has a higher range of motion in elbow flexion and in flexion, abduction, and, in particular, external rotation of the shoulder that throws the ball (Figure 1). Similar significant differences between groups were obtained by DTW (Figures 2 and 3) and RMSE analysis, mainly for right elbow flex $(\mathrm{RMSE}=3.354)$, right shoulder abduction $(\mathrm{RMSE}=7.221)$, and right shoulder flex $(\mathrm{RMSE}=2.04)$ movements.

The movements that occur in the trunk and pelvis presented two different phases: an anticipatory phase, where the trunk rotated to the same side as the arm (following the preparatory backswing of the arm component) and the pelvis moved in the opposite direction; and a second phase, on releasing the ball, where the trunk and pelvis moved in opposite directions. Group II had less trunk rotation during the movement than the healthy controls (Figure 4) and had increased lateral inclination of the trunk with posterior pelvic tilt during the first phase of the movement, followed by inversion of the lateral trunk inclination (to the opposite side) during the release of the ball (Figure 5). These differences of trunk and pelvis behavior can also be seen by analysis of the RMSE for thoracic rotation $(\mathrm{RMSE}=1.59)$ and pelvic forward tilt $(\mathrm{RMSE}=2.54)$ movements.

In the lower limbs, some differences between groups in flexion of the hip, knee, and ankle were also observed (Figure 6). Participants in Group II showed less variation in the range of movements that occur in the lower limbs, especially in the flexion of ankle and knee. During the throwing phase, participants in Group I increased their flexion by 10 degrees in both hips. Figures $7-10$ also show the same differences in motor patterns used by both groups for left hip flex $(\mathrm{RMSE}=3.34)$; left and right hip rotation $(\mathrm{RMSE}=2.20$ and 2.76, respectively); and left and right knee flex motion (RMSE $=2.94$ and 2.66, respectively).

Differences were also observed between the groups regarding the duration of the overarm throwing task (Table II). There was a significant difference in total movement duration $(p=0.001)$ as well as in the time spent during the anticipatory phase with a downward backswing movement $(p=0.004)$ and during the release of the ball $(p=0.031)$. There was not a significant correlation between chlorpromazine equivalent dose and full movement duration $(r=0.305 ; p=0.311)$, 


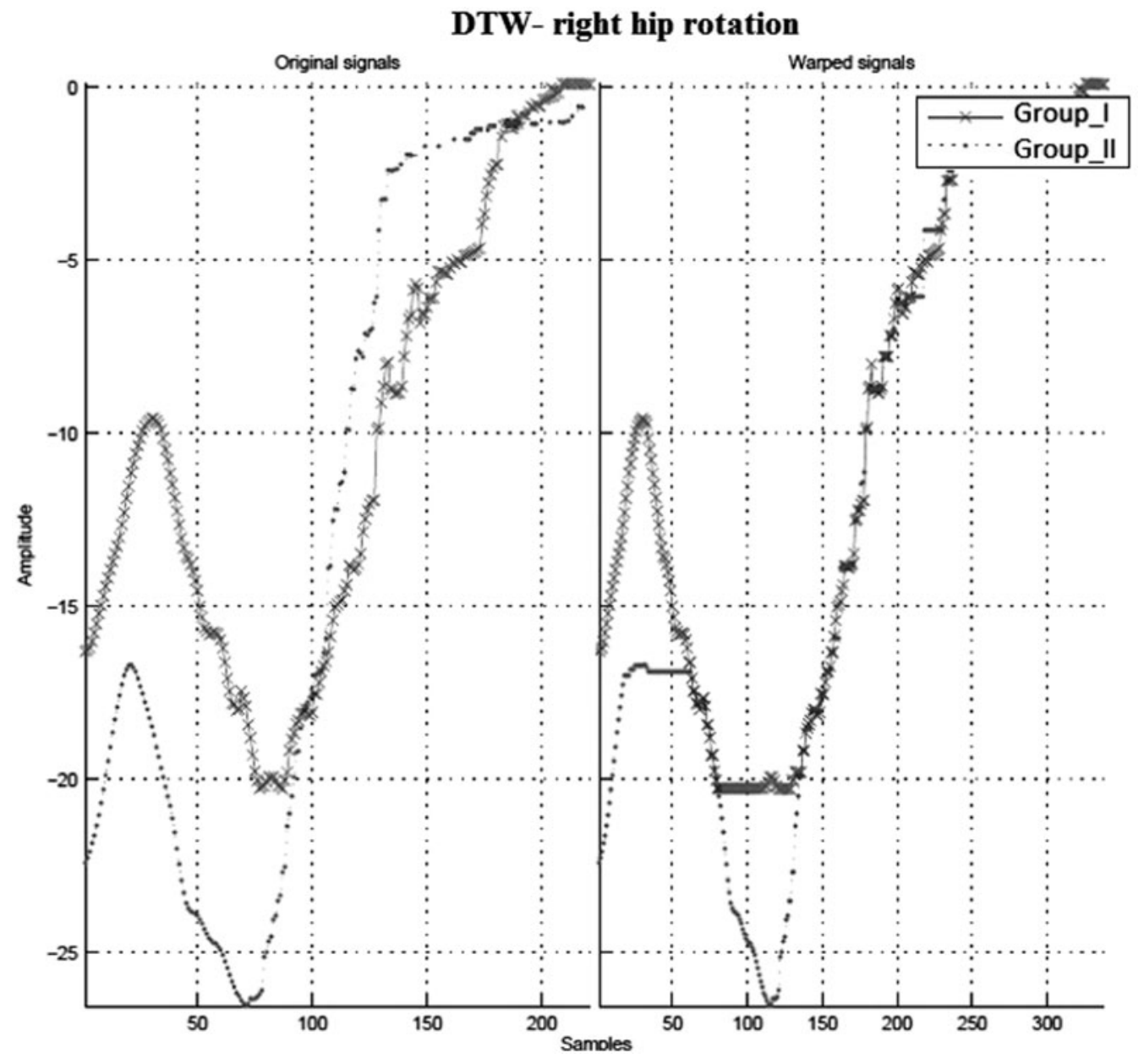

Figure 8. Dynamic time warping (DTW) algorithm computed for right hip rotation motion for both groups.

anticipatory phase duration $(r=-0.005 ; p=0.987)$, and release of the ball phase duration $(r=0.321 ; p=0.284)$, which suggests that participant performance was not influenced by antipsychotic medication.

\section{Discussion}

To our knowledge, this is the first study that analyzes kinematic parameters and motor patterns during a functional task in schizophrenia using a real-time system for motion capture. Two major findings were revealed in this investigation: (1) schizophrenia patients displayed a less developed movement pattern during performance of overarm throwing; and (2) the markerless system seems to have more daily life resemblance than clinical ratings.

The analysis of motion over time revealed that individuals with schizophrenia exhibited a low individualization of components, especially on the trunk and pelvis segments. Compared to healthy controls, schizophrenia patients compensated the rotation movement by a pattern of inclination and flexion movement at the level of the trunk and pelvis to increase strength for releasing the ball. This motor behavior is typically visible in younger children, but appears to be replaced by a more advanced pattern as they mature (Sparto et al. 2006; Haywood and Getchell 2009). According to Langendorfer and Roberton (2002), the rotation movement of the trunk is seen as a hallmark of the throwing movement and a control parameter for the change of the throwing pattern to a more developed state. There is also evidence that suggests that children stop performing a number of immature movements (e.g., trunk flexion) after six years of age, advancing to a more developed throwing movement sequence, which remains present in adulthood (van den Tillaar \& Ettema, 2009).

In the lower limbs, differences in motor patterns were noted between the two groups. In schizophrenia patients, the pelvis was further forward; there was more internal hip rotation, hip flexion, and knee flexion during the throwing motion compared to healthy controls. The increased involvement of the hip muscles by Group I can be related to lower pelvic stability. Analyzing the motor behavior of the lower limbs, it was noted that participants from both groups showed a different postural control strategy in response to upper limb movements. Participants from Group II activated the ankle strategy, which is used to respond to small disturbances in balance by action at the ankle joint. Group I, however, used the hip strategy, generally associated with larger or faster balance oscillation with a greater involvement of hip muscles (Runge et al. 1999). Because the motor task assessment required a short range of motion and a slow throw, schizophrenia patients exhibited less effective postural adjustment with the hip strategy to compensate for the movements that occurred in the upper limbs (Yiou et al. 2012). 


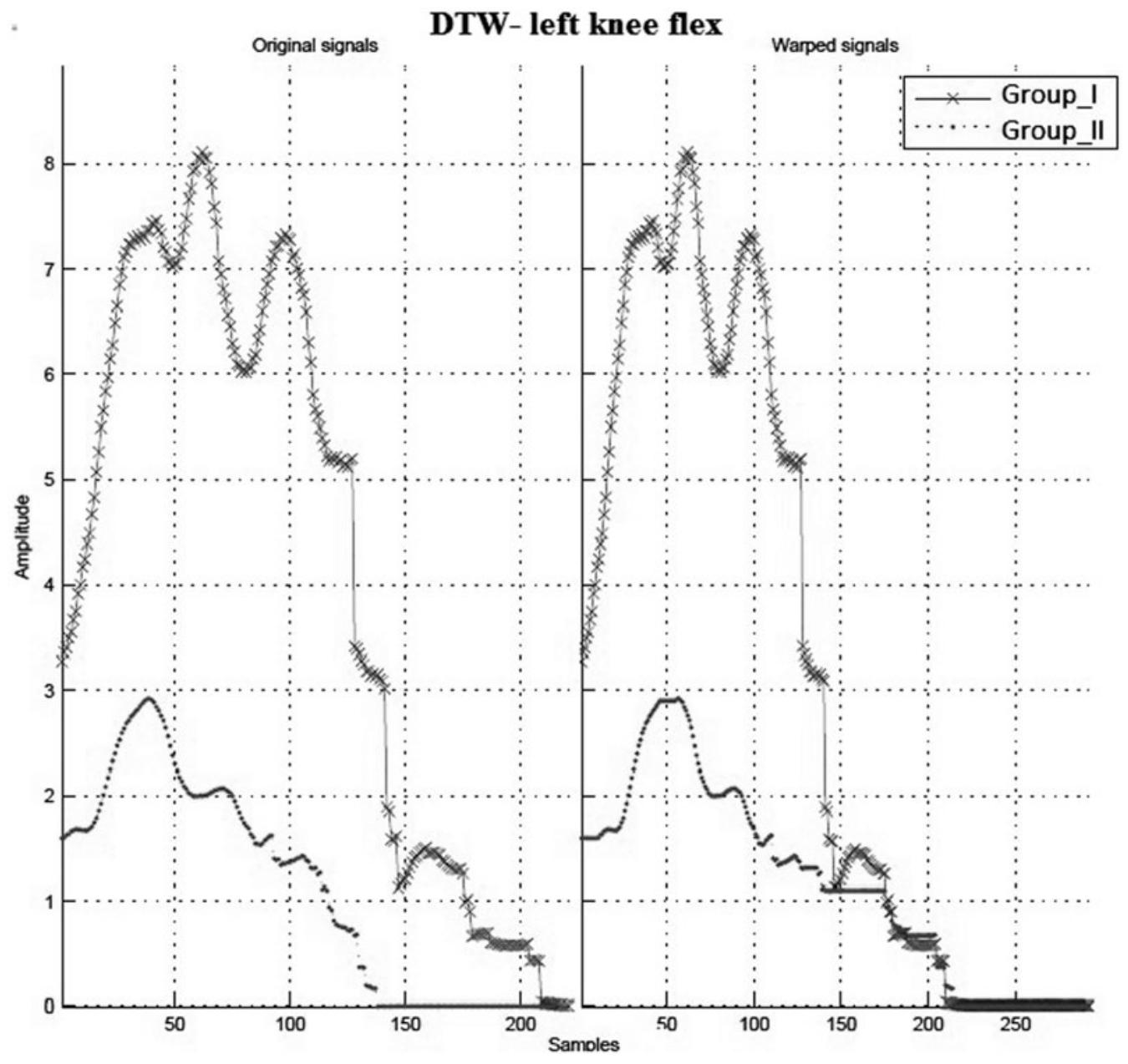

Figure 9. Dynamic time warping (DTW) algorithm computed for left knee flex motion for both groups.

Abnormal postural adaptation in schizophrenia patients was also found by Kent et al. (2012), who observed increased postural sway and less complex postural responses when the visual input was removed compared with participants from the control group. According to Girolami et al. (2010), anticipatory activation of the trunk muscles along with changes in the center of pressure are present in children with typical development as young as 7 years of age, and are sustained in adulthood.

Furthermore, participants with schizophrenia spent more time executing the motor task. This finding supports studies that showed a low reaction time and disturbances in initiation, intention, or willed actions in schizophrenia patients (Fuller et al. 1999; Cunnington et al. 2002).

Motor impairments should be interpreted as a sign of a neurological disorder, causing significant interference with the activities of daily living. In schizophrenia, motor coordination deficits have been reported as a significant neuro-developmental vulnerability marker for illness. Clinically, mild motor impairments are associated with NSS that were regarded as a more reliable reflection of the "integrity of the central nervous system" (Gillberg and Kadesjo 2003, p. 60). The evidence suggests that dysfunction in central mechanisms controlling movements largely contributes to impairment of motor behavior, expressed in inadequate coordination, timing of forces and muscle activity, sensory processing, and anticipatory adjustments (Ostensjo et al. 2004). In our study, the motor behavior of schizophrenia patients was characterized by these alterations that may represent latent neuro-developmental abnormality.

Subtle neurological signs do not only refer to trait-related characteristics such as neuro-developmental deficits (Patankar et al. 2012; Martins et al. 2013) or genetic liability (Niethammer et al. 2000; Gourion et al. 2004; Bachmann et al. 2005; Chan and Gottesman 2008), but are also associated with state-related changes such as acuity of psychopathological symptoms or treatment response (Bachmann et al. 2005). Hence, new reliable methods to automatically assess motor deficits in schizophrenia do not only facilitate the examination of motor changes but can also be of clinical importance for establishing acuity of the disease and prognosis.

Limitations of this exploratory research comprise the fact that schizophrenia patients were on antipsychotic treatment during the examination, which may have limited the genuine motor disturbance analysis. Participants' physical activity history was not considered and may also affect the performance on the assessed motor task. Reduced sample size and the use of non-probability sampling limited the power to detect significant differences and prevent results from being generalized to the wider population. Future investigations should continue to examine motor abnormalities in schizophrenia and their impact on social functioning and a patient's 


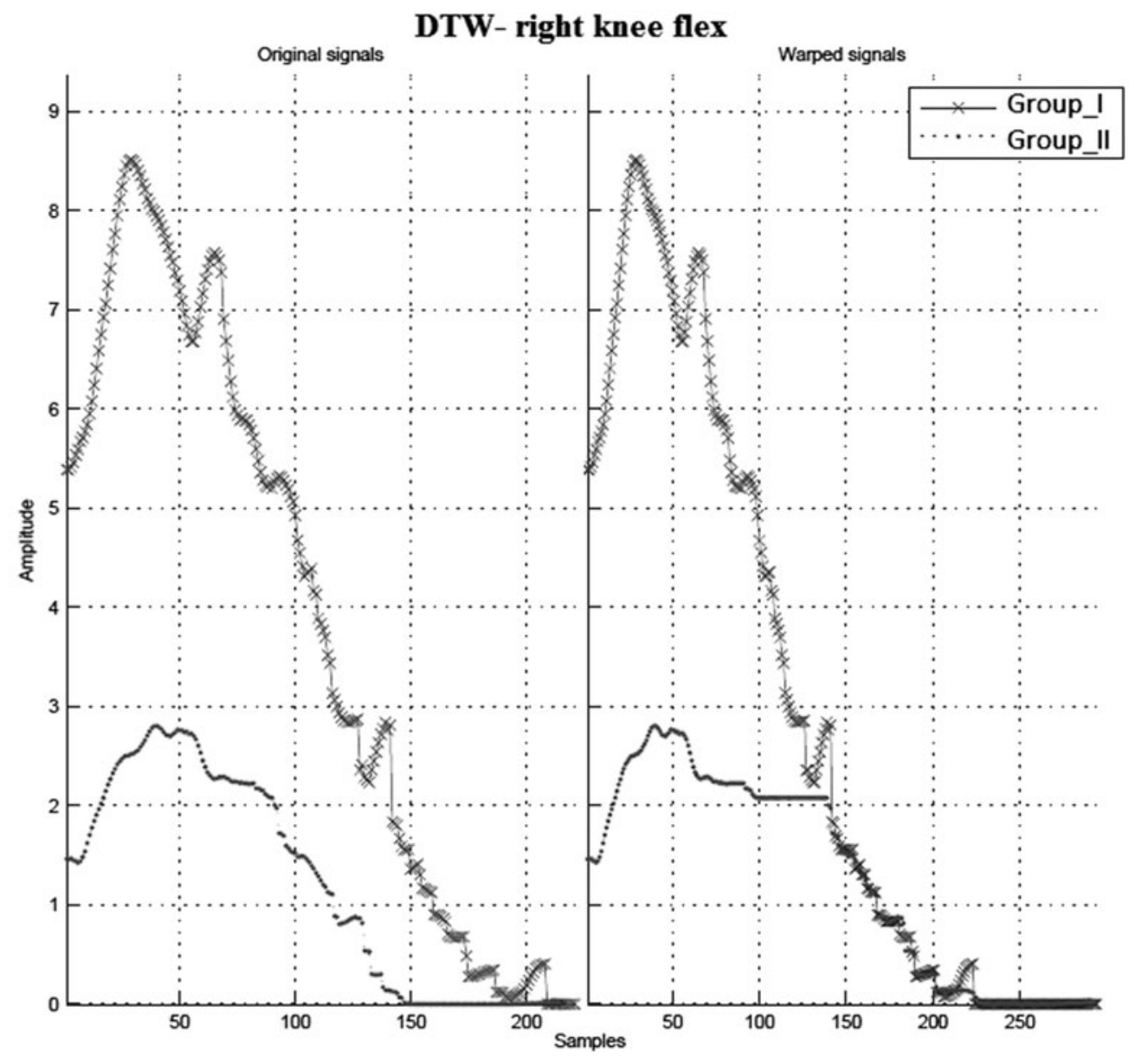

Figure 10. Dynamic time warping (DTW) algorithm computed for right knee flex motion for both groups.

Table II. Throwing movement duration for both groups.

\begin{tabular}{|c|c|c|c|c|c|}
\hline & \multicolumn{4}{|c|}{ Group } & \multirow[b]{3}{*}{$p$} \\
\hline & \multicolumn{2}{|c|}{$\mathrm{I}(n=13)$} & \multicolumn{2}{|c|}{ II $(n=16)$} & \\
\hline & $\mathrm{M}(\mathrm{SD})$ & Min-Max & $\mathrm{M}(\mathrm{SD})$ & Min-Max & \\
\hline Full movement duration (s) & $2.32(0.33)$ & $1.80-2.77$ & $1.81(0.27)$ & $1.34-2.26$ & $\leq 0.001^{\mathrm{a}}$ \\
\hline Anticipatory phase duration (s) & $1.24(0.30)$ & $0.78-1.74$ & $0.94(0.20)$ & $0.62-1.36$ & $0.004^{\mathrm{a}}$ \\
\hline Release of the ball phase duration (s) & $1.08(0.31)$ & $0.52-1.57$ & $0.87(0.19)$ & $0.58-1.32$ & $0.031^{\mathrm{a}}$ \\
\hline
\end{tabular}

${ }^{\mathrm{a}}$ Independent samples $t$-test.

quality of life. Longitudinal investigations with individuals at risk or in first episode patients using neuro-imaging techniques should also be developed.

Assessments of motor control using quantitative measurements provide objective evidence on motor disturbances in schizophrenia and further insight about pathophysiologic mechanisms of the disease. The study of motor dysfunction as an intrinsic part of schizophrenia seems to be crucial and also allows development of more effective practices in health and rehabilitation services.

\section{References}

Bachmann S, Bottmer C, Schroder J. 2005. Neurological soft signs in first-episode schizophrenia: A follow-up study. Am J Psychiatry 162(12):2337-2343.

Blondis TA, Snow JH, Accardo PJ. 1990. Integration of soft signs in academically normal and academically at-risk children. Pediatrics 85(3 Pt 2):421-425.

Bombin I, Arango C, Buchanan RW. 2005. Significance and meaning of neurological signs in schizophrenia: Two decades later. Schizophr Bull 31(4):962-977.

Bruininks RH. 1978. Bruininks-Oseretsky test of motor proficiency. Minnesota: American Guidance Service.

Buchanan RW, Heinrichs DW. 1989. The Neurological Evaluation Scale (NES): A structured instrument for the assessment of neurological 
signs in schizophrenia. Psychiatry Res 27(3):335-350.

Cannon M, Caspi A, Moffitt TE, Harrington H, Taylor A, Murray RM, Poulton R. 2002. Evidence for early-childhood, pan-developmental impairment specific to schizophreniform disorder: Results from a longitudinal birth cohort. Arch Gen Psychiatry 59(5):449-456.

Chan RCK, Gottesman II. 2008. Neurological soft signs as candidate endophenotypes for schizophrenia: A shooting star or a Northern star? Neurosci Biobehav Rev 32(5):957-971.

Clarke MC, Tanskanen A, Huttunen M, Leon DA, Murray RM, Jones PB, Cannon M. 2011. Increased risk of schizophrenia from additive interaction between infant motor developmental delay and obstetric complications: Evidence from a population-based longitudinal study. Am J Psychiatry 168(12):1295-1302.

Cunnington R, Windischberger C, Deecke L, Moser E. 2002. The preparation and execution of self-initiated and externally-triggered movement: A study of event-related fMRI. Neuroimage 15(2):373-385. doi: 10.1006/nimg.2001.0976.

Erlenmeyer-Kimling L, Rock D, Roberts SA, Janal M, Kestenbaum C, Cornblatt B, Adamo UH, Gottesman II. 2000. Attention, memory, and motor skills as childhood predictors of schizophrenia-related psychoses: The New York High-Risk Project. Am J Psychiatry 157(9):1416-1422.

Fuller R, Jahanshahi M. 1999. Concurrent performance of motor tasks and processing capacity in patients with schizophrenia. J Neurol Neurosurg Psychiatry 66(5):668-671.

Fuller R, Nathaniel-James D, Jahanshahi M. 1999. Movement-related potentials prior to self-initiated movements are impaired in patients with schizophrenia and negative signs. Exp Brain Res 126(4):545-555.

Gallahue D, Ozmun J. 2003. Compreendendo o desenvolvimento motor. Bebês, crianças, adolescentes e adultos. 3rd ${ }^{\mathrm{a}}$ ed. São Paulo; Brazil: Phorte Editora.

Gillberg C, Kadesjo B. 2003. Why bother about clumsiness? The implications of having developmental coordination disorder (DCD). Neural Plast 10(1-2):59-68.

Girolami GL, Shiratori T, Aruin AS. 2010. Anticipatory postural adjustments in children with typical motor development. Exp Brain Res 205(2):153-165.

Gourion D, Goldberger C, Olie JP, Loo H, Krebs MO. 2004. Neurological and morphological anomalies and the genetic liability to schizophrenia: A composite phenotype. Schizophr Res 67(1):23-31.

Haywood K, Getchell C. 2009. Life span motor development. 5th ed. Champaign, IL: Human Kinetics.

Jahn T, Cohen R, Hubmann W, Mohr F, Kohler I, Schlenker R, Niethammer R, Schroder J. 2006. The Brief Motor Scale (BMS) for the assessment of motor soft signs in schizophrenic psychoses and other psychiatric disorders. Psychiatry Res 142(2-3):177-189.

Kay SR, Fiszbein A, Opler LA. 1987. The positive and negative syndrome scale (PANSS) for schizophrenia. Schizophr Bull 13(2):261-276.

Kenney J, Keeping E. 1962. Root mean square in mathematics of statistics. 3rd ed. Princeton, NJ: Van Nostrand.

Kent JS, Hong SL, Bolbecker AR, Klaunig MJ, Forsyth JK, O'Donnell BF, Hetrick WP. 2012. Motor deficits in schizophrenia quantified by nonlinear analysis of postural sway. PLoS One 7(8):e41808.

Klausmann I. 2003. Kinematic analysis of fine motor performance in patients with schizophrenia and affective disorder five years after first hospitalization. Technische Universität München, Universitätsbibliothek.

Langendorfer SJ, Roberton MA. 2002. Individual pathways in the development of forceful throwing. Res Q Exerc Sport 73(3):245-256.

Manschreck T. 1992. Experimental analysis of motor phenomena in schizophrenia. In: Spitzer M, Uehlein F, Schwartz M, Mundt C, editors. Phenomenology, language \& schizophrenia. New York: Springer. pp 258-273.

Marques I, Catenassi F. 2005. Restrições da tarefa e padrões fundamentais de movimento: Uma comparação entre o chutar e o arremessar. R. da Educação Física/UEM 16(2):155-162.

Martins IP, Lauterbach M, Luis H, Amaral H, Rosenbaum G, Slade PD, Townes BD. 2013. Neurological subtle signs and cognitive development: A study in late childhood and adolescence. Child Neuropsychol 19(5):466-478. doi: 10.1080/09297049.2012.693911.

Moilanen K. 2011. Diagnostics and determinants of schizophrenia. The Northern Finland 1966 Birth Cohort Study. Oulu: University of Oulu,
Faculty of Medicine.

Müller M. 2007. Information retrieval for music and motion. Berlin: Springer.

Niethammer R, Weisbrod M, Schiesser S, Grothe J, Maier S, Peter U, Kaufmann C, Schröder J, Sauer H. 2000. Genetic influence on laterality in schizophrenia? A twin study of neurological soft signs. Am J Psychiatry 157(2):272-274.

Organic Motion. 2012. Achieving measurable advances in Life Science research and motion analysis. Retrieved 16 January 2012, from http:// www.organicmotion.com/

Ostensjo S, Carlberg EB, Vollestad NK. 2004. Motor impairments in young children with cerebral palsy: Relationship to gross motor function and everyday activities. Dev Med Child Neurol 46(9):580-589.

Patankar VC, Sangle JP, Shah HR, Dave M, Kamath RM. 2012. Neurological soft signs in children with attention deficit hyperactivity disorder. Indian J Psychiatry 54(2):159-165.

Putzhammer A, Perfahl M, Pfeiff L, Ibach B, Johann M, Zitzelsberger U, Hajak G. 2005a. Performance of diadochokinetic movements in schizophrenic patients. Schizophr Res 79(2-3):271-280.

Putzhammer A, Perfahl M, Pfeiff L, Muller JL. 2005b. [Threedimensional ultrasonic movement analysis of gait and handmovements in schizophrenic patients]. Psychiatr Prax 32(Suppl. 1):S38-S42. doi: 10.1055/s-2004-828491.

Putzhammer A, Klein HE. 2006. Quantitative analysis of motor disturbances in schizophrenic patients. Dialogues Clin Neurosci 8(1):123-130.

Roberton M, Halverson L. 1984. Developing children: Their changing movement. Philadelphia: Lea \& Febiger.

Runge CF, Shupert CL, Horak FB, Zajac FE. 1999. Ankle and hip postural strategies defined by joint torques. Gait Posture 10(2):161-170.

Sakoe H, Chiba S. 1978. Dynamic-programming algorithm optimization for spoken word recognition. IEEE Trans Acoust Speech Signal Processing 26(1):43-49.

Sparto PJ, Redfern MS, Jasko JG, Casselbrant ML, Mandel EM, Furman JM. 2006. The influence of dynamic visual cues for postural control in children aged 7-12 years. Exp Brain Res 168(4):505-516.

Tigges P, Mergl R, Frodl T, Meisenzahl EM, Gallinat J, Schroter A, Hegerl U. 2000. Digitized analysis of abnormal hand-motor performance in schizophrenic patients. Schizophr Res 45(1-2):133-143.

van den Tillaar R, Ettema G. 2009. Is there a proximal-to-distal sequence in overarm throwing in team handball? J Sports Sci 27(9):949-955.

Varambally S, Venkatasubramanian G, Gangadhar BN. 2012. Neurological soft signs in schizophrenia-The past, the present and the future. Indian J Psychiatry 54(1):73-80.

Varlet M, Marin L, Raffard S, Schmidt RC, Capdevielle D, Boulenger JP, Bardy BG. 2012. Impairments of social motor coordination in schizophrenia. PLoS One 7(1):e29772.

Veselinović T, Schorn H, Vernaleken I, Schiffl K, Hiemke C, Zernig G, Gur R, Gründer G. 2011. Effects of antipsychotic treatment on psychopathology and motor symptoms. A placebo-controlled study in healthy volunteers. Psychopharmacology 218(4):733-748.

Walther S, Strik W. 2012. Motor symptoms and schizophrenia. Neuropsychobiology 66(2):77-92.

Wechsler D. 1997. Escala de inteligência de Wechsler para adultos -

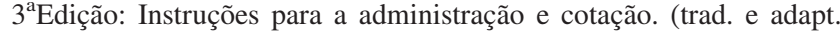
Portuguesa CEGOC-TEA, 2006). Lisboa: CEGOC-TEA.

Welham J, Isohanni M, Jones P, McGrath J. 2009. The antecedents of schizophrenia: A review of birth cohort studies. Schizophr Bull 35(3):603-623. doi: 10.1093/schbul/sbn084.

Woods SW. 2003. Chlorpromazine equivalent doses for the newer atypical antipsychotics. J Clin Psychiatry 64:663-667.

Wolff AL, O'Driscoll GA. 1999. Motor deficits and schizophrenia: The evidence from neuroleptic-naive patients and populations at risk. J Psychiatry Neurosci 24(4):304-314.

Yiou E, Caderby T, Hussein T. 2012. Adaptability of anticipatory postural adjustments associated with voluntary movement. World $\mathrm{J}$ Orthop 3(6):75-86. 